\title{
Precision Full-Wave Rectifiers with Current Active Elements and Current Biasing
}

\author{
L. Langhammer and J. Jerabek
}

\begin{abstract}
This article discusses universal precision rectifiers using current active elements and current sources for diode excitation. The paper introduces a circuit solution of the universal precision full-wave rectifier with intention to reduce the negative effect of diode reverse recovery time. Furthermore, experimental results are given and a comparison of the new circuit of precision full-wave rectifier and its known variant is presented.
\end{abstract}

Keywords - Current conveyor, Diode reverse recovery time, Rectifiers, UCC

\section{INTRODUCTION}

Precision rectifiers are important building blocks for signal processing and instrumentation of low level signals. The basic problem of conventional precision rectifiers based on diodes and operational amplifiers is that during the transition of diodes from their non-conduction state to their conduction state the operational amplifiers have to recover with a finite small signal, which leads to a significant distortion during zero crossing of the input signal. [1]

Recently, articles describing circuits using active elements operating in a current or mixed mode have appeared. We can mention as examples current conveyors (CC) [2-5], operational transconductance amplifiers (OTA) [6], current followers (CF) [7], digitally adjustable current amplifiers (DACA) $[8,9]$ and their multiple-output variants such as UCC $[3,4,5]$, MOTA [10, 11], MO-CF [11], etc. Using these elements instead of the common operational amplifiers, we can obtain wider bandwidth, improve the signal-to-noise ratio, achieve a higher precision of the output rectified signal and decrease the energy consumption.

The paper [12] describes the bridge rectifier with two conveyors connected as a voltage-to-differential current converter. The issue of temperature sensitivity and sensitivity to small changes of voltage biasing are also solved. Rectifiers which use for their function current followers and operate in the current mode are described in [7]. In this case, a double output current follower (DOCF) and four diodes are used. The problem of small input signals

Manuscript received March 25, 2013

L. Langhammer Faculty of Electrical Engineering and Communication, Brno University of Technology, Technicka 12, 61600 Brno, Czech Republic (e-mail: xlangh01@stud.feec.vutbr.cz)

J. Jerabek Faculty of Electrical Engineering and Telecommunication, Brno University of Technology, Technicka 12, 61600 Brno, Czech Republic (e-mail: jerabekj@feec.vutbr.cz) can be solved by employing an additional voltage follower on the input of the circuit, which provides the high input impedance of the described rectifier. Furthermore, it is possible to come across rectifiers consisting of operational transconductance amplifiers in [6]. The bandwidth of presented circuit is $50 \mathrm{kHz}$. This circuit does not use diodes and is based on four or five OTA.

We can find one of the basic scheme of full-wave precision rectifier in $[1,13]$. The described circuit is the highfrequency precision rectifier consisting of two secondgeneration current conveyors connected in a form of differential voltage-to-current converter and uses four diodes for its function. In case of small signals at zero crossing transition portion, when the diodes are closed, the differential voltage-to-current converter turns into a high gain voltage differential amplifier. A modified circuit of the high-frequency precision rectifier using the current biasing for diodes proposed in [12] is described in [14]. Another possible solution of the precision full-wave rectifier can be found for example in [15-18].

\section{EFFECT OF THE DIODE REVERSE RECOVERY TIME ON PRECISION RECTIFICATION}

One of the major factors limiting the use of diodes for highfrequency signals is the diode reverse recovery time. This time can be described as the transition time of the diode from its open state to the closed state. [14, 19] A simple subcircuit with two diodes, which is shown in Fig. 1) is used for the majority of precision rectifier circuits using current diode excitation.

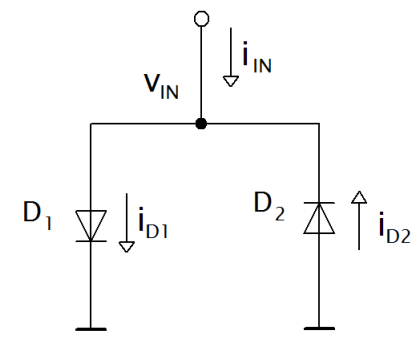

a)

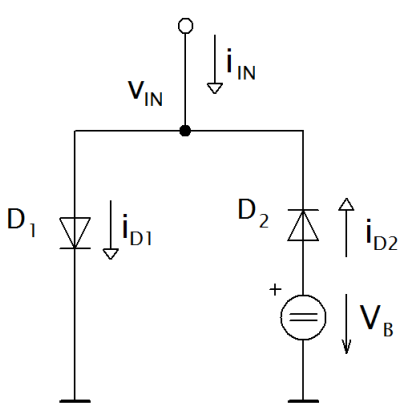

b)

Fig. 1. Subcircuit with current excited diodes b) subcircuit with bias 
When the polarity of the input current is changing the diode stays for a short time in the conductive state even during the opposite polarity, which results in an overshoot of the diode current into the reverse polarity, as shown in Fig. 2). Rising frequency increases the negative effect of the diode reverse recovery time and this leads to increasing distortions of rectified signal. This is due to the fact the semiconductor diode is a non-ideal component. The duration of this period depends on the physical parameters of the diodes respectively on the material from which the diode is made and on its technological performance. Each $\mathrm{P}$ or $\mathrm{N}$ type of semiconductor contains not only majority carriers but also minority carriers of the opposite polarity. P-N junction remains open for these minority carries during the reverse polarity, therefore, the diode still conducts current until the exhaustion of the minority carriers and then the reverse current decreases to a minimum value.

The diode reverse recovery time $t_{\mathrm{k}}$ can be divided into periods $t_{\mathrm{s}}$ and $t_{\mathrm{d}}$. During the period $t_{\mathrm{s}}$ the minority carriers are discharged from $\mathrm{P}-\mathrm{N}$ junction and an almost constant voltage remains on the diode. This voltage is slightly smaller than the forward voltage. This period is followed by the period $t_{\mathrm{d}}$. In time $t_{\mathrm{d}}$, the parasitic capacitance of diodes is being charged. After this time, the voltage on the diode remains at the value of the reverse voltage. We can reduce the time $t_{\mathrm{s}}$ by increasing the reverse current flowing through the diode, but this causes a greater overshoot when the polarity changes. [14]

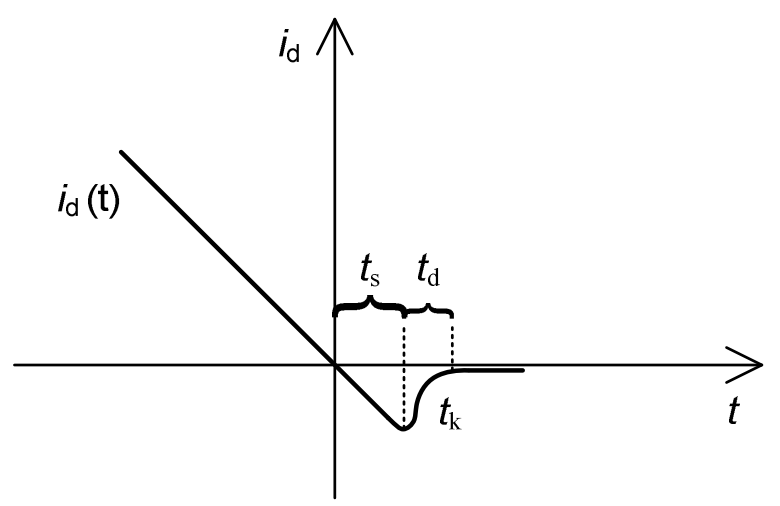

Fig. 2. Detail of the diode current $I_{\mathrm{d}}$ during zero crossing of the input signal

We can obtain a shorter time $t_{\mathrm{d}}$ by modification of the circuit, when we add a voltage source $V_{\mathrm{B}}$ as it can be seen in Fig. $1 \mathrm{~b}$ ). This modification set diodes during the zero crossing of the input current at the border of their conductive state, thereby it reduces the changes of the voltage at the input node when the input current changes. However, higher bias results into increasing of the current which flows through diodes around the region where the input current changes its polarity. Therefore, the value of the bias for diodes must be set as a compromise between overshoot to opposite polarity of the current flowing through diodes and increasing of the diode zero crossing input current.

\section{UNIVERSAL PRECISION FULL-WAVE RECTIFIER WITH CURRENT BIASING}

\section{A. Description of the Universal Precision Full-Wave Rectifier}

One of the basic precision rectifier circuits which can be found for example in [20] can be seen in Fig. 3 a). The circuit consists of a half-wave rectifier based around the first operational amplifier and a summing amplifier formed by the second operational amplifier. The half-wave rectified current flows through $\mathrm{D}_{2}$ on a positive half cycle of the input signal $v_{\mathrm{IN}}$. This signal is summed in the summing amplifier with the input signal having relative weights set according to values of resistors $R_{4}=R / 2$ and $R_{1}=R_{2}=R_{3}=R_{5}=R$ resulting in a full-wave rectified signal on the output. Generally, this type of circuit works well at low frequencies, but produces a large waveform distortion at frequencies higher than $1 \mathrm{kHz}$. This is due to the fact that at the transition point of the input signal, the diodes are closed and the operational amplifier operates in an open-loop configuration. As the input signal frequency increases, limited slew rate more prevents the OA from switch diodes rapidly which leads to a distortion of the output signals. [20]

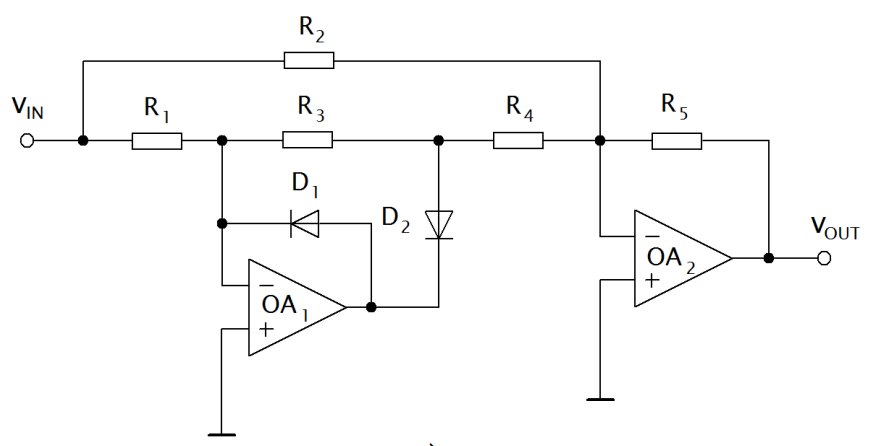

a)
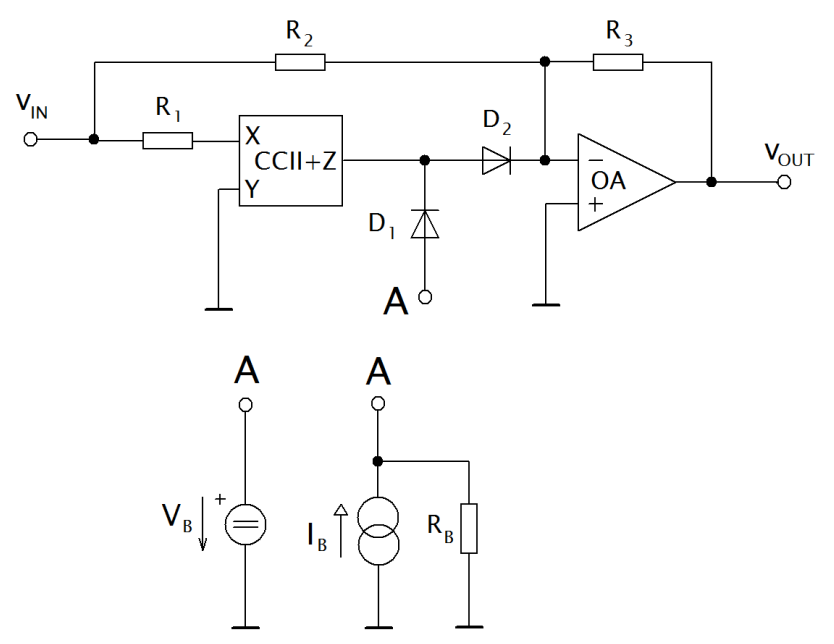

b)

Fig. 3. Universal precision full-wave rectifier a) based on operational amplifiers, b) based on current conveyor and a voltage or current source for bias

This issue can be solved by circuit modifications from Fig. $3 \mathrm{~b}$ ), when a part of the circuit which operates as the half-wave rectifier is replaced by one with a larger bandwidth. This can be achieved by replacing the operational amplifier by a second generation current conveyor. The high output impedance of the current 
conveyors helps overcome the turn-ON resistance of the diodes, so the circuit operates at higher frequencies. Signal weights are again set according to the values of resistors when $R_{2}=R_{3}=R$ and $R_{1}=R / 2$ which gives a full-wave rectified signal on the output. As can be seen in Fig. 3 b), the circuit with the current conveyor is supplemented with a voltage source or current source and resistor for biasing to set the diodes close to their open state.

The transfer function for this circuit is given by

$v_{\text {OUT }}=-\left|v_{I N}\right|$,

when

$R_{1}+r_{X}=\frac{R}{2}$

where $r_{\mathrm{X}}$ is the equivalent resistance at the current input of the current conveyor.

In case when the voltage source is used to bias the diodes, the circuit is temperature-sensitive. Another disadvantage of a rectifier with this type of biasing is its sensitivity to small variations of bias voltage which are displayed and the offset voltage appears at the output. A higher temperature stability can be achieved by using a DC current source to bias the diodes. The output offset voltage is easily controlled and adjusted to its minimal level. [12] Therefore, the next proposal considers using current sources to bias diodes.

\section{B. Proposal and Experimental measurements}

The proposed circuit can be seen in Fig. 4. It uses the design of a circuit modification for bias taken from [12] and modified for universal precision full-wave rectifier. The modification involves an addition of two current sources, two diodes, and two operational amplifiers, where $\mathrm{OA}_{2}$ is working as a voltage follower and $\mathrm{OA}_{3}$ as a current-tovoltage converter. This circuit works properly only in voltage mode. When the $\mathrm{OA}_{3}$ is omitted simulations show the circuit is supposed to work also in a mixed and current mode. The bias current flows through the diodes $\mathrm{D}_{3}$ and $\mathrm{D}_{4}$ and produces a voltage drop across them. This voltage is then fed into a voltage follower. Residual current flowing through $\mathrm{D}_{1}$ and $\mathrm{D}_{2}$ creates a voltage drop across these diodes and as a result the diodes are set close to the open state. The current is then diverted through another current source to ground, so it is possible to set the zero offset of the output signal.

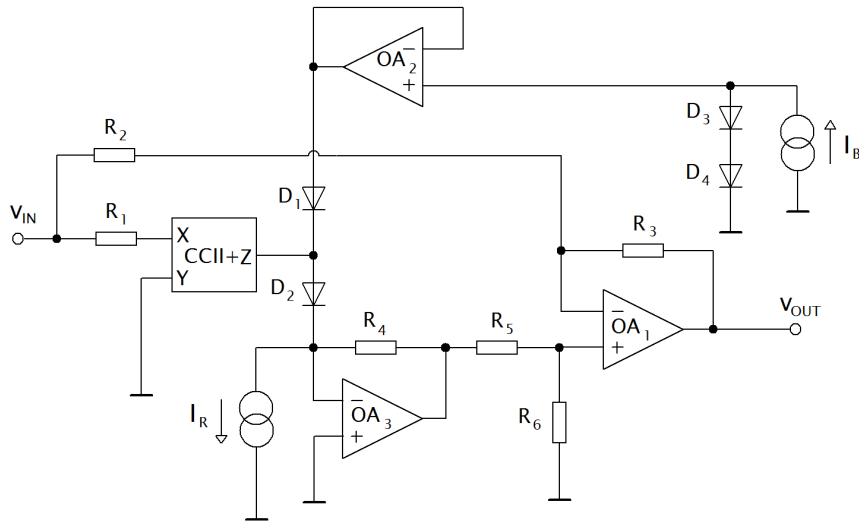

Fig. 4. Proposed circuit of the universal precision full-wave rectifier with current biasing
The summing operational amplifier is replaced by a differential amplifier to achieve a proper operation of the circuit. Resistances are set $\mathrm{R}_{2}=\mathrm{R}_{3}=\mathrm{R}_{4}=\mathrm{R}_{5}=\mathrm{R}_{6}=\mathrm{R}$ and $\mathrm{R}_{1}=\mathrm{R} / 2$. This circuit was implemented in the form of printed circuit board and experimental measurements were carried out to verify its function. For the implementation the transconductance operational amplifier OPA861 [21] was used as the current active element and THS4052C [22] as the operational amplifier, diodes of type 1N4148 and resistors of values $1 \mathrm{~K} \Omega$ and $470 \Omega$ were used. For illustration a comparison of the basic universal precision full-wave rectifier from Fig. $3 \mathrm{~b}$ ) and the proposed circuit is presented. For the basic circuit a voltage source of value $V_{\mathrm{B}}=0.6 \mathrm{~V}$ is used to bias the diodes. In case of the proposed circuit, the biasing current $I_{\mathrm{B}}$ and residual current $I_{\mathrm{R}}$ are both set to $40 \mu \mathrm{A}$. In Fig. 5 a) shows the output rectified signals for the input signal frequency of $1 \mathrm{MHz}$ and the amplitude of $1 \mathrm{~V}$. Figure $5 \mathrm{~b}$ ) shows a comparison of output rectified signals with the same input frequency when the amplitude of the input signal was $0.2 \mathrm{~V}$. From the output waveforms it can be seen that the proposed circuit achieves significantly better results for signals with smaller amplitudes than the basic circuit.

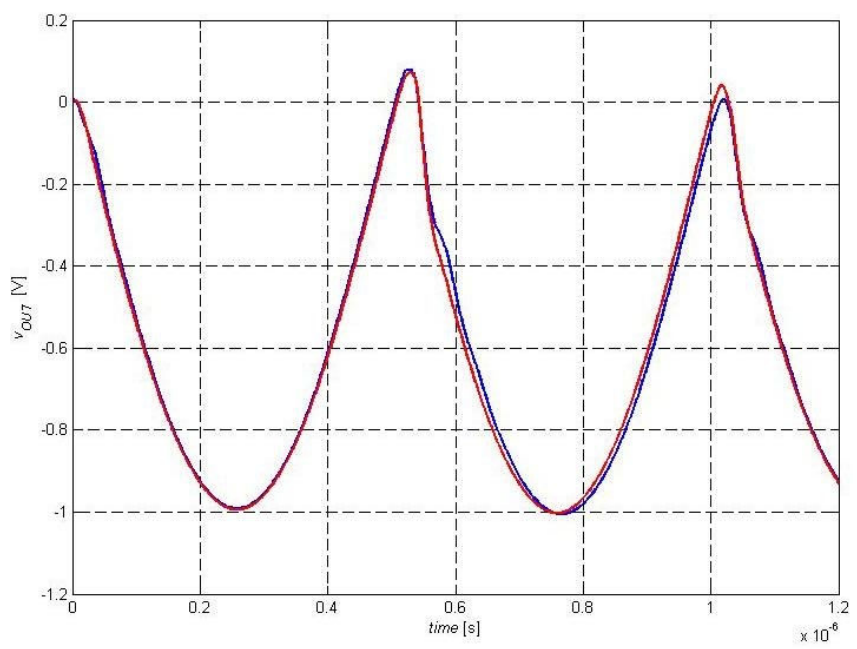

a)

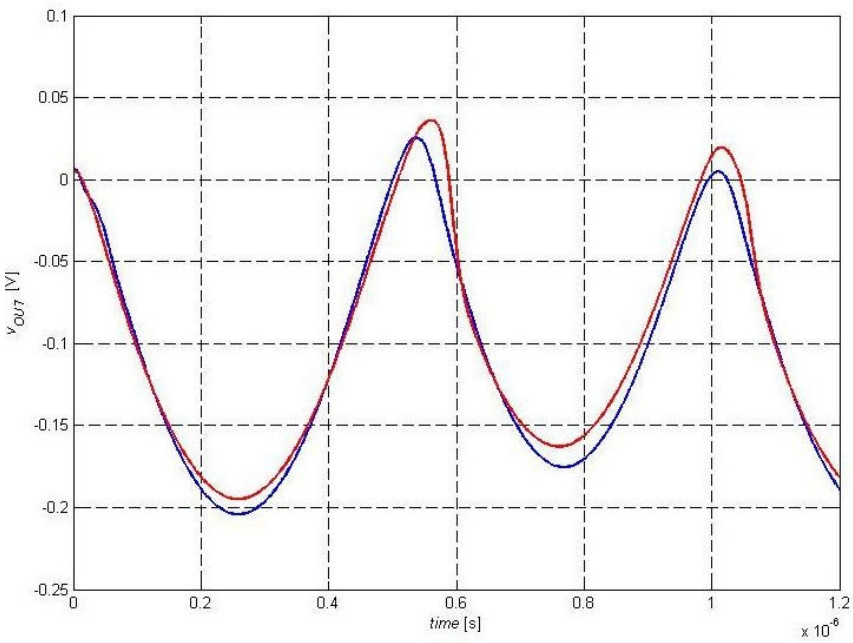

b)

Fig. 5. Measured output waveforms of proposed circuit (blue line) and basic circuit from fig. $3 \mathrm{~b}$ (red line) when input signal frequency was $1 \mathrm{MHz}$ and amplitude a) $1 \mathrm{~V}$, b) $0.2 \mathrm{~V}$ 
Fig. 6) shows a comparison of the transfer function of implemented circuits. As can be seen the transfer function does not follow the expected gradient during the positive half-cycle due to the parasitical resistance $r_{X}$ of the conveyor's current input. This negative effect is not so significant for the proposed circuit.

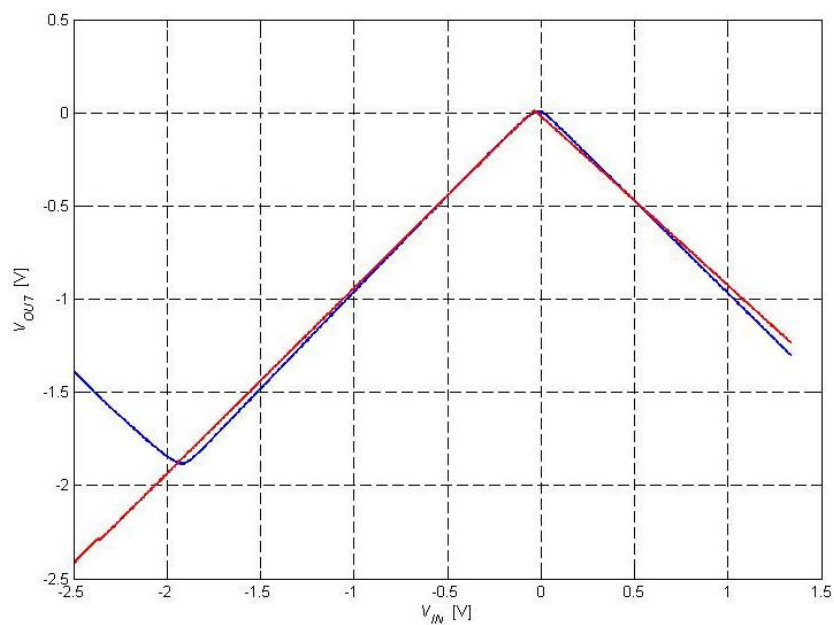

Fig. 6. Measured DC transfer functions proposed circuit (blue line) and basic circuit from Fig. 3b (red line)

From Fig. 7) it is possible to compare the details of the transfer function of the proposed circuit when using current diode biasing and without bias. This picture shows that the output offset can be set to a minimum value using the current sources to bias the diodes.

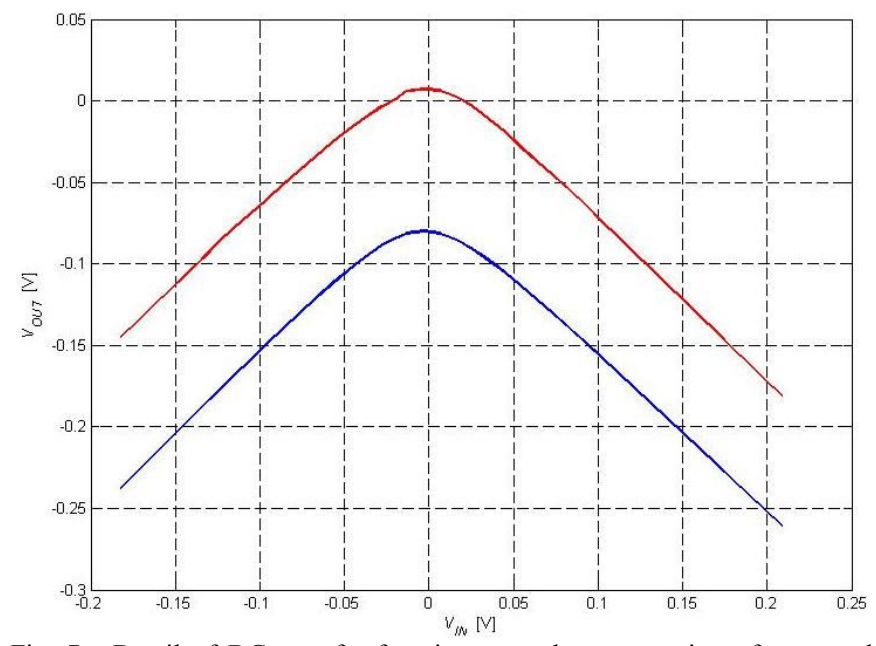

Fig. 7. Detail of DC transfer function around zero crossing of proposed circuit with bias (red line), without bias (blue line)

In conclusion, the DC value transfer $p_{\mathrm{DC}}$ has been analyzed to compare the accuracy of circuits. Results are based on simulations of these circuits when the input signal frequency was in the range from $10 \mathrm{kHz}$ to $1 \mathrm{MHz}$ and the amplitude of the input signal was $100 \mathrm{mV}$. From Fig. 8 can be seen that the proposed circuit gives better results when the frequency is increasing. The DC value transfer is given by (3) [23]

$p_{D C}=\frac{\int_{T} y_{R}(t) d t}{\int_{T} y_{I D}(t) d t}$,

where $y_{\mathrm{R}}(\mathrm{t})$ represents the actual rectifier signal, $y_{\mathrm{ID}}(\mathrm{t})$ represents the ideally rectified signal and $T$ is the period of the input signal.

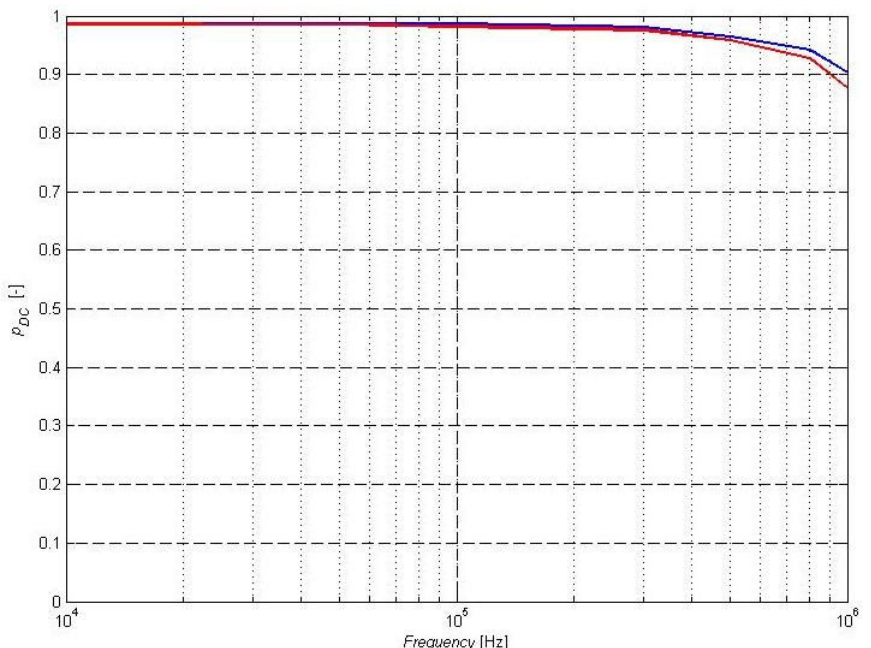

Fig. 8. Measured DC value transfer $p_{\mathrm{DC}}$ proposed circuit (blue line) and basic circuit from Fig. 3h (red line)

\section{CONCLUSION}

The aim of this work is to propose a circuit solution of the universal precision full-wave rectifier with current sources to bias diodes with an effort to limit the effect of the diode reverse recovery time. The function of the proposed circuit was verified by experimental measurements. The paper compares measured rectified output waveforms, measured DC transfer functions of implemented circuits and DC value transfer $p_{\mathrm{DC}}$ based on simulations.

Using the modification of the biasing solution presented in [12] wider bandwidth has been achieved by reducing distortions caused by the negative effect of the diode reverse recovery time. Another advantage of proposed circuit is that the output offset can be easily controlled and set to its minimum level. Better results of the small signal rectification than in case of the basic circuit with a voltage source to bias diodes are also obtained.

\section{REFERENCES}

[1] TOUMAZOU C., LIDGEY F. J., CHATTONG S :High frequency current conveyor precision full-wave rectifier. IEE 1994, Electronics Letters Online No: 19940539.

[2] LIU S.I., WU D. S., TSAO H. W., WU J., TSAY J. H.: Nonlinear circuit applications with current conveyors. IEE PROCEEDINGS-G, Vol. 140, No. 1, FEBRUARY 1993.

[3] SPONAR R., VRBA K.: Measurements and Behavioral Modeling of Modern Conveyors. IJCSNS International Journal of Computer Science and Network Security, VOL.6 No.3A, March 2006.

[4] VRBA, K., JERABEK J.: Selected features of the universal current conveyor, example of the application design. Elektrorevue, 24.10.2006, č. 41, s. ISSN 1213-1539.

[5] JERABEK, J.; VRBA, K. SIMO type low-input and high-output impedance current- mode universal filter employing three universal current conveyors. AEU - International Journal of Electronics and Communications, 2010, vol. 64, no. 6, pp. 588-593

[6] JONGKUNSTIDCHAI C., FONGSAMUT C., KUMWACHARA K. SURAKAMPONTORN W. : Full-wave rectifiers based on operational transconductance amplifiers. C. Jongkunstidchai et al. / Int. J. Electron. Commun. (AEÜ) 61 (2007) 195 - 201.

[7] TILIUTE D. E.: Full-wave current-mode precision rectifiers using unity-gain cells. Electronics and Electrical Engineering.- Kaunas: Technologija, 2003. - No. 7(49). - P. 26-29.

[8] JERABEK J., SOTNER R., VRBA K., KOUDAR I.: Fully differential versatile adjustable filter with current active elements. Elektrorevue, 2010/7 - 15. 2. 2010, ISSN 1213 - 1539

[9] JERABEK, J.; KOTON, J.; SOTNER, R.; VRBA, K. Adjustable band-pass filter with current active elements: two fully-differential and single- ended solutions. ANALOG INTEGRATED CIRCUITS AND SIGNAL PROCESSING, 2013, vol. 74, no. 1, pp. 129-139. 
[10] JERABEK J., VRBA K.: Novel Universal Filters Using Only Two Current Active Elements, In Proc of Third Int Conf on Systems (ICONS'08), IEEE Computer Society, Cancun, pp. 285-289, 2008.

[11] JERABEK J., VRBA K., JELINEK M.: Versatile adjustable filter with current followers transconductance amplifiers and a minimum number of components. Elektrorevue, 2010/46 - 2. 7. 2010, ISSN $1213-1539$.

[12] WILSON B., MANNAMA V.:Current-mode rectifier with improved precision. IEE 1995, Electronics Letters Online No: 19950185.

[13] HAYATLEH K., PORTA S. LIDGEY F. J.:Temperature independent current conveyor precision rectifier. IEE 1994, Electronics Letters Online No: 199414.54.

[14] KUBANEK D., VRBA K.: Precision rectifiers with current excitation of diodes and the effect of the diode reverse recovery time to their function. Elektrorevue, 2010/6 - 15. 2. 2010, ISSN 1213 - 1539.

[15] KOTON J., HERENCSAR N., VRBA K., CICEKOGLU O.: Versatile Precision Full-Wave Rectifier Using Current and Voltage Conveyor, Latest Trends on Circuits, Systems and Signals, ISSN: 1792-4324.

[16] BURIAN J., KOTON J., HERENCSAR N.: Universal Voltage Conveyor and Current Conveyor in Fast Full-Wave Rectifier, The International Journal of Advances in Telecommunications, Electrotechnics, Signals and Systems Vol 1, No 2-3, pp. 37-41, 2012, doi: 10.11601/ijates.v1i2-3.8, ISSN: 1805-5443.

[17] KOTON J., HERENCSAR N., VRBA K.: Current and Voltage Conveyors in Current and Voltage-Mode Precision Full-Wave Rectifiers, RADIOENGINEERING, VOL. 20, NO. 1, APRIL 2011.

[18] KOTON J., HERENCSAR N., VRBA K.: Precision Full-wave Rectifier Using Current Conveyors and Two Diodes, ICN 2012 : The Eleventh International Conference on Networks, ISBN: 978-1-61208183-0.

[19] LANGHAMMER, L. Non-linear circuits using current active elements. Brno University of Technology, Faculty of Electrical Engineering and Telecommunication, 2012. 66 s. Supervisor of the master's thesis Ing. Jaroslav Koton, Ph.D..

[20] GIFT S. J. G., MAUNDY B.: Versatile precision full-wave rectifiers for instrumentation and measurements, Manuscript received December 7, 2005; revised May 3, 2007. Digital Object Identifier 10.1109/TIM.2007.904565.

[21] Texas Instruments - OPA861 - Wide bandwidth Operational Transconductance Amplifier (OTA) (datasheet). Online, < http://www.ti.com/product/opa861 >

[22] Texas Instruments - THS4052 - 70-MHz high-speed Amplifiers (datasheet). Online, $<$ http://www.ti.com/product/ths $4052>$

[23] D. Biolek, V. Biolkova, and Z. Kolka, "AC Analysis of Operational Rectifiers via Conventional Circuit Simulators," WSEAS Transactions on Circuits and Systems, vol. 3, no. 10, pp. 22912295, 2004 